\title{
Interpretation of a selection plateau through complex segregation analysis: Euclidean distance index selection for flower colour in the Davis population of Gerbera
}

\author{
KENNETH R. TOURJEE*, JAMES HARDING \& THOMAS G. BYRNE \\ Department of Environmental Horticulture, University of California, Davis, CA 95616-8587, U.S.A.
}

\begin{abstract}
Two cycles of recurrent phenotypic selection with a weighted Euclidean distance index based on flower colour were applied to individual plants sampled from the Davis population of gerbera (Gerbera jamesonii H. Bolus ex Hooker). The index $(I)$ included CIELAB $(1976)$ variables hue $(H)$, chroma $(C)$ and value $(L)$. The narrow-sense heritability $\left(h^{2}\right)$ for $I$ in cycle 0 was estimated at more than 1.0 by half-sib family means and consequently overestimated the selection response. However, the genetic variance was exhausted after two cycles of selection and genetic correlations among the component traits of the index became increasingly positive. Complex segregation analysis (CSA) of index scores in the Davis population supports a mixed Mendelian model with a completely dominant segregating major gene accounting for 20 per cent of the phenotypic variance. This model estimates $h^{2}=0.9$ for $I$ and is discussed as it pertains to the effects of selection on the genetic parameters of the selected population.
\end{abstract}

Keywords: chroma, genetic correlation, heritability, hue, quantitative traits, value of colour.

\section{Introduction}

Flower colour may be treated as a composite of three metric characters: hue, chroma and value (McGuire, 1992; Voss, 1992). These characters describe the type (namely red, yellow, green or blue, etc.), intensity and lightness of colour, respectively. Their distributions in the Davis population of gerbera indicate that they are continuous variables (Tourjee et al., 1993). The variability described by these distributions is largely under genetic control (Tourjee et al., 1995) and subject to selection. Therefore, these component traits permit the precise definition of colour phenotypes and are amenable to the procedures of biometrical genetics.

The genetic gain to be made under a recurrent phenotypic selection regime depends on the genetic architecture of the trait in a given population. Selection will cause only a temporary change in genetic variance owing to genetic disequilibrium and gain will continue if a quantitative trait is determined by an infinite number of unlinked polygenic loci (Bulmer, 1971). A selection plateau may result from limits imposed by the nature of the trait (namely percentages have limits of 0

\footnotetext{
${ }^{*}$ Correspondence.
}

and 100 , and physiological processes operate within biological constraints), reduced fertility in selected lines, dominant favourable alleles, overdominance or the effects of concurrent natural selection (Falconer, 1989). In finite populations a selection plateau may be reached if genetic variance is exhausted. Genetic variance may be exhausted through selection or drift in small populations especially when genes of large effect contribute to genetic variability. Hospital \& Chevalet (1993) used simulation studies to show that when genetic assumptions are not restricted to the infinitesimal model selection theory overestimates response, and Chevalet (1994) described a finite genetic model for estimating response to selection. Complex segregaton analysis (CSA) (Elston, 1993) determines if the infinitesimal model is appropriate for a trait and provides insights into the success of long-term selection experiments.

The purpose of this study was to investigate the response to recurrent phenotypic selection of flower colour in the Davis population of gerbera using a weighted Euclidean distance index (Harding et al., 1991). The index was used to minimize distance to a target colour of specified hue $(H)$, chroma $(C)$ and value $(L)$. The selection results are discussed in relation to CSA of the index scores. 


\section{Materials and methods}

\section{Base population}

Gerbera (Asteraceae) is a heterozygous, crosspollinated cut-flower crop. The Davis population has been randomly mated and grown as an annual in the same greenhouse for 16 generations. Plants in $7.6 \mathrm{~L}$ pots were randomly assigned to locations within the greenhouse in each generation. In most generations 40 parents have been selected from approximately 400 plants $(n=454$ in generation 15$)$ and intermated in a disconnected factorial mating design (Comstock \& Robinson, 1948). The 40 parents were mated via four $5 \times 5$ crossing blocks, yielding 25 full-sib families per block in generation 15. Previous selection in the population has been based on a desired gains index (Pesek \& Baker, 1969) that included flower yield and flower dry weight, but not flower colour. This population and its colour traits have been described more fully elsewhere (Byrne et al., 1977; Harding et al., 1991; Tourjee et al., 1993; Yu et al., 1993).

\section{Colour measurement}

Colour measurements of flowers were obtained using the Minolta (Ramsey, New Jersey) CR-200 chroma meter. It is a tri-stimulus colour analyser for measuring the reflective colours of surfaces. The CR-200 has an $8 \mathrm{~mm}$ diameter aperture with diffuse illumination from a pulsed xenon arc lamp and a $0^{\circ}$ viewing angle. Measurements were made using the $H$ and $C$ transformations of the CIELAB (1976) colour space as described by Tourjee et al. (1993).

\section{Index}

Euclidean distance indices provide a convenient means to measure the distance of individuals from a reference point. A Euclidean distance index scores phenotypes relative to an optimal phenotype that may be intermediate in value. Index scores can be used to select individuals to minimize mean distance from a target colour. The index used in this study contained only the three colour traits, $H, C$ and $L$, as follows:

$I=\left[\frac{1}{2}(\Delta H)^{2}+\frac{1}{4}(\Delta C)^{2}+\frac{1}{4}(\Delta L)^{2}\right)^{1 / 2}$.

The target colour was $H=85, C=80$ and $L=50$; which can be described as a deep yellow colour. Individual scores were obtained by taking the difference between the reference point and observation for each trait, and then calculating the Euclidean distance as described in the equation. There were no plants in the unselected population that matched the target colour, although co-ordinates of the target were within the range of each of the component traits. Trait $H$ was weighted twice as much as $C$ or $L$ because hue was deemed more important to flower quality than chroma or value.

\section{Selection and mating scheme}

Twelve plants with the lowest index scores were selected from generation 15 of the Davis population (cycle 0 ). These plants were intermated in a $6 \times 6$ factorial mating design to obtain cycle 1 with a population size of 246 plants. The procedure was repeated on cycle 1 to produce cycle 2 , a population of 261 plants. The selection intensity was 2.3 and 2.0 standard deviation units for each cycle of selection, respectively (Falconer, 1989).

In cycles 1 and 2 the plants were grown in $15 \mathrm{~cm}$ pots until first flower when their flower colour was measured. Plants were discarded after first flower if they were not selected as parents. Cycle 1 was grown in the autumn of 1991, cycle 2 in the spring of 1992.

All estimates of cycles 0,1 and 2 genetic parameters are based on half-sib family means using least squares estimates as described in Weber \& Wricke (1986). Cycle 0 consisted of four subpopulations (one from each crossing block); therefore, the estimates given for cycle 0 represent the average of four estimates. Statistical calculations to obtain relevant mean squares and mean products were performed using the GLM procedure of sAS (SAS Institute, 1988). The limiting value of heritability $\left(h_{\mathrm{L}}^{2}\right)$, resulting from the offsetting processes of genetic variance reduction from selection and regeneration from recombination, was estimated according to Gomez-Raya \& Burnside (1990):

$h_{\mathrm{L}}^{2}=\frac{-1+\sqrt{1+4 h_{0}^{2} k\left(1-h_{0}^{2}\right)}}{2 k\left(1-h_{0}^{2}\right)}$,

where $k$ is the selection intensity in standard deviation units and $h_{0}^{2}$ is the narrow-sense heritability in cycle 0 . The inbreeding effective population number $\left(N_{\mathrm{e}(\mathrm{f})}\right)$ was calculated according to Crow \& Kimura (1970). Estimates of skewness and kurtosis were made using the Univariate Procedure of sAs (SAS Institute, 1985).

\section{Complex segregation analysis}

CSA utilizes pedigree analysis (Cannings et al., 1978; Elston \& Stewart, 1971) to determine the underlying genetic structure of a trait. Regressive models (Bonney, 1984) have been formulated to facilitate CSA. Briefly, these models define residuals from major gene effects that are treated as dependent random variables. These 
residuals can be interpreted in terms of polygenic and environmental factors by regressing the residuals of an individual on those of antecedent members of the pedigree. The application of CSA to a plant population was described by Tourjee et al. (1995).

This investigation used the REGC program's class D model of the Statistical Analysis for Genetic Epidemiology (SAGE) Release 2.1 computer software package (Elston, Department of Biometry and Genetics, Louisiana State University Medical Center, New Orleans, LA) which is based on a regressive model as described in Tourjee et al. (1995). CSA estimates were not produced on cycle 0,1 or 2 owing to limitations of the segregation analysis algorithm. Therefore, a separate random sample (CSA sample) was taken of pedigrees from the Davis population to estimate genetic parameters of the selected population. Each pedigree was composed of a half-sib family (parents and offspring) that contained from one to five full-sib families representing three different generations of the base population. The number of individuals per full-sib family ranged from two to six. The index scores of the same individuals $(n=467)$ from the Davis population of gerbera analysed for hue in Tourjee et al. (1995) were pooled with those of additional individuals derived from the Davis population. This yielded a total of 673 individuals partitioned into 37 pedigrees for the CSA.

Restricted models representing various transmission hypotheses were defined that allowed for a mixture of as many as three normal phenotypic distributions (see Tourjee et al., 1995 for details). The three distributions can be related to unobservable types that may or may not show genetic inheritance. Each type is composed of two discrete factors, $A$ or $a$, and is denoted as either $A A, A a$ or $a a$. Three arbitrary transmission parameters

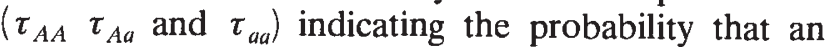
individual of a given type transmits factor $A$ to an offspring were also estimated in the general model. Parameters for modelling these distributions are defined in Table 1. Low index score is associated with the $a$ factor,

Three means $\left(\mu_{A A}, \mu_{A a}, \mu_{a a}\right)$ were estimated with a common variance assumed for the phenotypic distributions. The parameter $\mu_{A a}$ was forced to equal $\mu_{A A}$ or $\mu_{a a}$ (complete dominance), to be midway between $\mu_{\mathrm{AA}}$ and $\mu_{\mathrm{aa}}$ (additivity) or to be independent (namely arbitrary placement allowing for partial or overdominance). Residual (namely after adjusting for the major gene effect) nonindependence among relatives, parent-offspring and sib-sib, is parameterized by $\rho_{\mathrm{po}}$ and $\rho_{\mathrm{ss}}$, respectively. Residual heritability is estimated as $2 \rho_{\mathrm{po}}$. All model parameters were estimated by using maximum likelihood procedures. Optimization of the
Table 1 List of parameters available in the REGC program for CSA used in this investigation

\begin{tabular}{|c|c|}
\hline Parameter & Description \\
\hline$q_{a}$ & $\begin{array}{l}\text { Frequency of allele } a \text { at a single locus with } \\
A \text { and } a \text { alleles }\end{array}$ \\
\hline$\tau_{i}$ & $\begin{array}{l}i=A A, A a \text { or } a a ; \text { transmission probability } \\
\text { for } A A, A a \text { and } a a \text { genotypes }\end{array}$ \\
\hline$\mu_{i}$ & $\begin{array}{l}i=A A, A a \text { or } a a ; \text { mean for } A A, A a \text { or } a a \\
\text { genotypes }\end{array}$ \\
\hline$\sigma_{\mathrm{rph}}^{2}$ & Residual $^{\mathrm{z}}$ phenotypic variance \\
\hline$\rho_{i}$ & $\begin{array}{l}i=\mathrm{fm}, \text { po or ss; residual }{ }^{\mathrm{z}} \text { correlation between } \\
\text { mates, parent-offspring, and between } \\
\text { siblings, respectively }\end{array}$ \\
\hline
\end{tabular}

${ }^{\mathrm{z}}$ residual refers to effects remaining after adjustment for the major gene.

likelihood functions was achieved through the complete direct search method.

An overall heritability $\left(h_{\Gamma}^{2}\right)$ was estimated from CSA that includes the additive effects of both major- and polygenes. The additive variance estimate for the major gene (Falconer, 1989) was calculated from $V_{\mathrm{a}}=2 p q[a+d(q-p)]^{2}$. The polygene additive variance estimate was obtained as the product of $\sigma_{\mathrm{rph}}^{2}$ and $2 \rho_{\mathrm{po}}$. The sum of the major- and polygene additive variances was then expressed as a ratio of this sum over the phenotypic variance of the CSA sample.

The likelihood ratio test (LRT) was used to compare restricted models against the general model (Edwards, 1978). The LRT, twice the negative $\log _{e}$ likelihood $(-2 \ln L)$ of each model, is constructed as the difference between the unrestricted and restricted models. It asymptotically approaches a $\chi^{2}$ distribution with d.f. equal to the difference in number of parameters between the two competing hierarchical models. Akaike's information criterion (AIC), $-2 \ln L+2$ (number of parameters), was used to compare nonhierarchical models (Akaike, 1974). The model providing the smallest AIC is the most appropriate for a given data set.

Three criteria for inferring the presence of a segregating major gene must be met by CSA to guard against the false detection of such a gene (Demenais et al., 1986). First, the transmission probability estimates of the general model must approximate Mendelian expectations. Secondly, a Mendelian model must not be rejected in comparison with the general model. Finally, a non-Mendelian model must not provide a better fit to the data set. Strategies for inferring genetic 
control of phenotypic variability are discussed more fully by Khoury et al. (1993).

\section{Results}

The frequency distributions of index scores for each cycle are given in Fig. 1. The index scores in cycle 0 ranged from 13.0 to 64.4 with a mean score estimate of 38.6 and variance $=85.01$. The distribution was approximately normal (skewness $=-0.15$, kurtosis $=$ 0.52 ). After two cycles of selection scores ranged from 10.4 to 31.7 with a mean score estimate of 14.6 and variance $=8.88)$. The cycle 2 distribution deviated from normality (skewness $=2.49$, kurtosis $=9.01$ ). The harmonic mean estimate of $N_{\mathrm{e}(f)}$ for two cycles of selection was 13.7 .

The means of the component traits and index scores for each cycle of selection are shown in Table 2. The means for $H$ and $C$ move closer to their target while $L$ moves further from its target. The minimum index score in the cycle 2 population had coordinates $H=83, C=74$ and $L=70$.

The narrow-sense heritability estimates for the component colour traits and the index are provided in Table 3. They are relatively high in cycle 0 and drop rapidly in two cycles of selection. The index heritability estimates for each cycle were 1.4, 0.4 and 0.0 , respectively.

The additive genetic correlations are not stable over the selection cycles (Table 4). The correlation between $H$ and $L$ is strongest and remains constant through two cycles of selection. The correlations between $H$ and $C$, and $C$ and $L$ are weaker but appear to change in a positive direction with selection.

The CSA sample had estimated mean index score $=36.9, \quad$ variance $=89.87, \quad$ skewness $=-0.151$ and kurtosis $=0.210$. The estimates (and SE) of transmission probabilities for the general model, $\tau_{A A}=1.0$, $\tau_{A a}=0.6 \pm 0.14$ and $\tau_{a a}=0.09 \pm 0.05$, approximate Mendelian expectations (Table 5). Since the $\tau_{A A}$ estimate was fixed at a boundary the d.f. in the LRT were reduced by one (Khoury et al., 1993). The bestfitting models were the arbitrary and dominant models, the dominant model being more parsimonious (Table
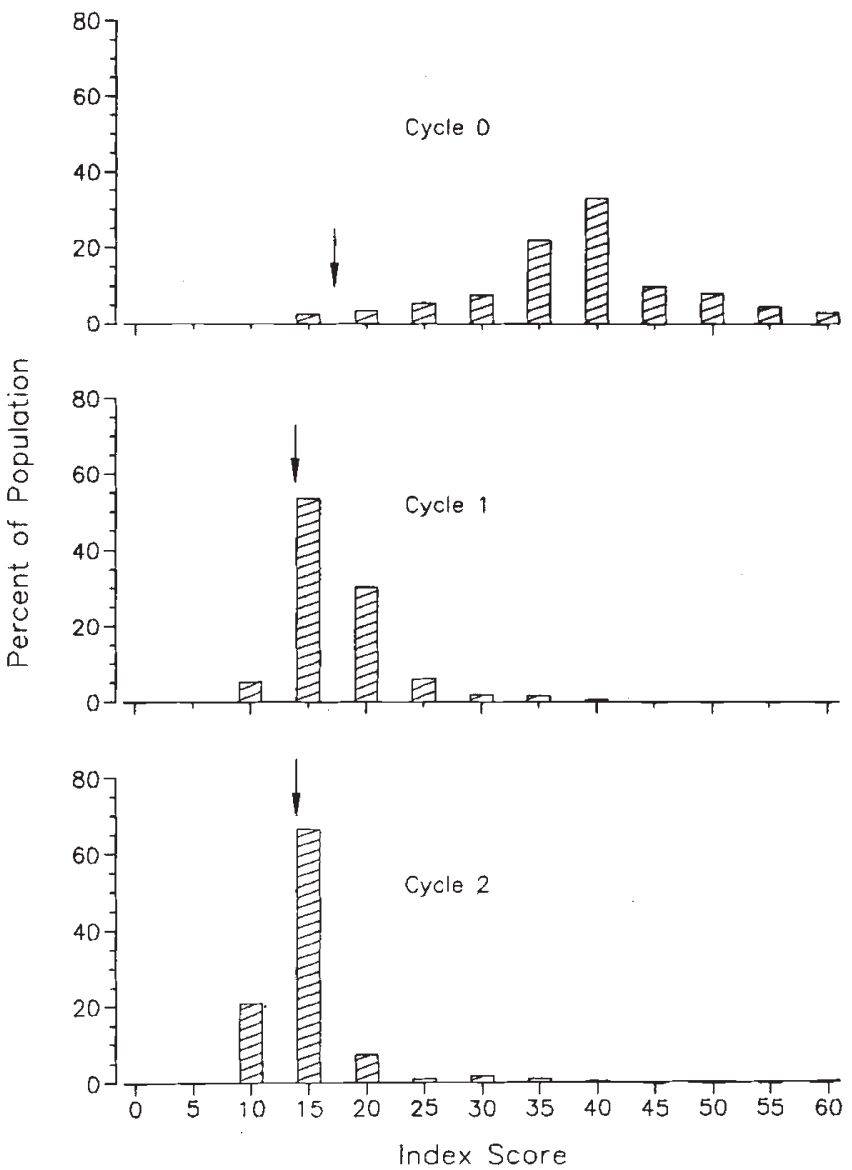

Fig. 1 Frequency distributions for the index score for phenotypes of cycle $0(n=454)$, cycle $1(n=246)$ and cycle $2(n=267)$ of selection applied to gerbera from the Davis population. The arrows indicate the location of the selection point $(17.2,13.9$ and 14.0 , respectively) imposed in each cycle.

Table 2 Mean estimatesand standard errors of component traits and index scores for each selection cycle $\left(n_{\mathrm{c} 0}=454, n_{\mathrm{c} 1}=246, n_{\mathrm{c} 2}=261\right.$ plants $)$ applied to gerbera from the Davis population

\begin{tabular}{ccccc}
\hline & \multicolumn{4}{c}{ Mean estimates $\pm(\mathrm{SE})$} \\
\cline { 2 - 5 } Cycle & Value $(L)$ & Chroma $(C)$ & Hue $(H)$ & Index $(I)$ \\
\hline 0 & $47.8 \pm(0.69)$ & $57.6 \pm(0.15)$ & $35.5 \pm(0.15)$ & $38.6 \pm(0.08)$ \\
1 & $74.8 \pm(0.57)$ & $68.8 \pm(0.66)$ & $68.8 \pm(0.66)$ & $17.6 \pm(0.30)$ \\
2 & $72.8 \pm(0.39)$ & $74.1 \pm(0.46)$ & $79.3 \pm(0.46)$ & $14.6 \pm(0.19)$ \\
Target & 50.0 & 80.0 & 85.0 & 0.0 \\
\hline
\end{tabular}


Table 3 Narrow-sense heritability estimates for selection cycles applied to gerbera from the Davis population

\begin{tabular}{lcccc}
\hline & \multicolumn{4}{c}{ Narrow-sense heritability estimates } \\
\cline { 2 - 5 } Sample & $L$ & $C$ & $H$ & $I$ \\
\hline Cycle 0 & 1.0 & 0.8 & 0.6 & 1.4 \\
Cycle 1 & 0.4 & 0.4 & 0.4 & 0.4 \\
Cycle 2 & 0.1 & 0.1 & 0.2 & 0.0 \\
\hline
\end{tabular}

Heritability estimates of cycles 0,1 and 2 were based on halfsib family means using least squares estimates.

Table 4 Additive genetic correlation estimates between hue and chroma $(H: C)$, hue and value $(H: L)$ and chroma and value $(C: L)$ for each selection cycle applied to gerbera from the Davis population

\begin{tabular}{llcr}
\hline & \multicolumn{3}{c}{ Additive genetic correlation estimates } \\
\cline { 2 - 4 } Cycle & $H: C$ & $H: L$ & $C: L$ \\
\hline 0 & 0.1 & 0.8 & -0.5 \\
1 & 0.5 & 1.0 & 0.4 \\
2 & 0.7 & 0.9 & 0.2 \\
\hline
\end{tabular}

5). A putative dominant major gene (favourable recessive allele) is identified with homozygote means of the index estimated at 23.1 and 39.3 , and a recessive allele frequency estimate of 0.34 (Table 6). Comparing the CSA sample variance with $\sigma_{\mathrm{rph}}^{2}$ indicates that the major locus accounts for approximately 20 per cent of the phenotypic variation of index scores (Table 6). The additive genetic variance of the major locus $(13.62)$ is

Table 6 Parameter estimates and their standard errors for the dominant and arbitrary models based on the CSA sample of gerbera from the Davis population

\begin{tabular}{ccc}
\hline & \multicolumn{2}{c}{ Estimates $( \pm \mathrm{SE})$} \\
\cline { 2 - 3 } Parameter & Dominant model & Arbitrary model \\
\hline$q_{a}$ & $0.34 \pm(0.05)$ & $0.32 \pm(0.05)$ \\
$\mu_{a a}$ & $23.1 \pm(1.53)$ & $23.3 \pm(1.41)$ \\
$\mu_{A a}$ & $39.3 \pm(0.77)$ & $40.7 \pm(1.23)$ \\
$\mu_{A A}$ & $39.3 \pm(0.77)$ & $37.3 \pm(1.53)$ \\
$\sigma_{\mathrm{rph}}^{2}$ & $71.9 \pm(8.52)$ & $71.7 \pm(8.18)$ \\
$\rho_{\mathrm{fm}}$ & $0.03 \pm(0.10)$ & $0.00 \pm(0.10)$ \\
$\rho_{\mathrm{po}}$ & $0.45 \pm(0.05)$ & $0.45 \pm(0.05)$ \\
$\rho_{\mathrm{ss}}$ & $0.59 \pm(0.06)$ & $0.60 \pm(0.05)$ \\
\hline
\end{tabular}

See Table 1 for parameter key.

Table 5 Likelihood ratio test (LRT) for genetic models of index scores in the CSA sample of gerbera from the Davis population $(n=673 ; 37$ pedigrees $)$

\begin{tabular}{|c|c|c|c|c|c|c|}
\hline Model & $\begin{array}{l}\text { Parameters } \\
\text { iterated }\end{array}$ & $-2 \ln L$ & d.f. & $\chi^{2}$ & $P$-value & AIC \\
\hline $\mathrm{GSL}^{2}$ & $\mu_{A A}, \mu_{A a}, \mu_{a a}, \sigma_{\mathrm{rph}}^{2}$ & 4523.4 & 4 & 76.6 & $<0.001$ & 4535.4 \\
\hline No major gene ${ }^{y}$ & $\begin{array}{l}\rho_{\mathrm{fm}}, q_{\mathrm{a}} \\
\mu, \sigma_{\mathrm{rph}}^{2}, \rho_{\mathrm{fm}}, \rho_{\mathrm{po}} \\
\rho_{\mathrm{ss}}\end{array}$ & 4488.9 & 5 & 42.02 & $<0.001$ & 4498.9 \\
\hline Env. ${ }^{x}$ & $\mu, \sigma_{\mathrm{rph}}^{2}, \rho_{\mathrm{fm}}$ & 4685.8 & 7 & 239.0 & $<0.001$ & 4691.8 \\
\hline Arbitrary $^{w}$ & $\begin{array}{l}\mu_{A A}, \mu_{A a}, \mu_{a a}, \sigma_{\mathrm{rph}}^{2} \\
\rho_{\mathrm{fm}}, \rho_{\mathrm{p} o}, \rho_{\mathrm{ss}}, q_{a}\end{array}$ & 4450.0 & 2 & 3.17 & 0.205 & 4466.0 \\
\hline Dominant $^{v}$ & $\begin{array}{l}\mu_{A A}, \mu_{a u}, \sigma_{\mathrm{rph}}^{2}, \rho_{\mathrm{fm}} \\
\rho_{\mathrm{po}}, \rho_{\mathrm{ss}}, q_{a}\end{array}$ & 4451.6 & 3 & 4.74 & 0.192 & 4465.6 \\
\hline Additive $^{v}$ & $\begin{array}{l}\mu_{A A}, \mu_{a a}, \sigma_{\mathrm{rph}}^{2}, \rho_{\mathrm{fm}} \\
\rho_{\mathrm{po}}, \rho_{\mathrm{ss}}, q_{a}\end{array}$ & 4477.2 & 3 & 30.18 & $<0.001$ & 4491.2 \\
\hline General $^{\mu}$ & $\begin{array}{l}\mu_{A A}, \mu_{A a}, \mu_{a a}, \sigma_{\mathrm{rph}}^{2} \\
\rho_{\mathrm{fm}}, \rho_{\mathrm{po}}, \rho_{\mathrm{ss}}, q_{a} \\
\tau_{A A}, \tau_{A a}, \tau_{a a}\end{array}$ & 4446.8 & & & & \\
\hline
\end{tabular}

See Table 1 for parameter key.

${ }^{2} \mathrm{GSL}$ is the general single locus (Mendelian) model allowing no residual correlation among relatives.

'No major gene' model assumes only polygenic and environmental variation.

${ }^{x}$ Env. model permits only environmental variation.

${ }^{\text {w}}$ Arbitrary model assumes both polygenic and major locus effects (mixed), and does not restrict the mean of the heterozygote.

"Mixed model with the heterozygote mean appropriately restricted.

"General model obtains MLE for all $\tau_{i}\left(\tau_{A A}=1.0, \tau_{A a}=0.6, \tau_{a a}=0.09\right)$ and serves as the comparison in the LRT. 
obtained from estimates of the allele frequency and genotypic means. $h_{\mathrm{T}}^{2}$ was estimated as 0.9. As the CSA sample represents an unselected population $h_{\mathrm{T}}^{2}$ can substitute for the cycle 0 half-sib family means estimate. $h_{\mathrm{L}}^{2}=0.77$ for the index (using $k=2.15$ and $h_{0}^{2}=0.9$ ) indicating that substantial genetic gain should be expected for several generations of phenotypic selection given the assumptions of the infinites mal model.

\section{Discussion}

The genetic architecture of a trait plays an important role in determining its response to selection. This response can be studied in terms of the change in its mean and variance, as well as covariances with other traits. The classical polygenic model assumes a large number of independent genes, each with small effect. This predicts that selection does not plateau, genetic variances remain unchanged (after reaching a limiting value) and genetic correlations become more negative (Bulmer, 1971; Lerner, 1950). If these predictions prove inaccurate the assumptions of the model need to be examined. CSA provides a mechanism to test hypotheses concerning the genetic basis of a trait, and to elucidate a model that more realistically fits the data.

The rapid change in the mean index score estimate, from 38.6 to 15.1 in two cycles of selection, is predicted by the high heritability estimate for the index score in the cycle 0 population (Table 3 ). This heritability estimate also predicts that the target would be reached in two cycles of selection. However, most of the gain was realized in the first cycle of selection with only modest gains made in cycle 2 . Inbreeding is not an important cause of drift for $N_{\mathrm{e}(\mathrm{f})}=13.7$ (Baker \& Curnow, 1969).

The mean estimates of $C$ and $H$ (cycle 2, 74.1 and 79.3, respectively) rapidly approach their targets through selection as predicted by their heritabilities (Table 2). However, the mean estimate for trait $L$ (cycle 2, 72.8), which also has a high heritability, does not approach its target score of 50, but moves further from it. This can be explained by the strong positive additive genetic correlation between $H$ and $L$, and the location of the target colour which resulted in $H$ and $L$ being selected in opposite directions. Placing a greater weight on $H$ than $L(2: 1)$ ensured that $H$ would reach its target and $L$ would not.

The Gomez-Burnside formula, given the usual assumptions of quantitative genetics, predicts that the heritability of the index score should decrease to a limiting value of 0.77 under selection. The dramatic drop in the heritability estimate of the index after two cycles of selection indicates that this prediction is greatly biased. Much of the additive genetic variance was captured in the first cycle of selection, and the remaining additive genetic variance was a small component of the phenotypic variance. This outcome is supported by the results of CSA which demonstrated the presence of a gene with large effect. The mean of cycle 2 suggests that the favourable allele of this gene was fixed. Consequently, the heritability estimate derived from the cycle 1 population, not that derived from cycle 0 , should be used to predict accurately the gain to be made in cycle 2 .

All three criteria for detecting a major gene through complex segregation analysis were met in this investigation (Table 5). The CSA dominant model's overall heritability estimate $(0.9)$ for the index of the unselected sample is more reasonable than that of the half-sib family means based on the least squares estimate (1.4). This is borne out in Table 2 by the gain realized from one cycle of selection. The CSA heritability of 0.9 and selection intensity of 2.3 standard deviation units should yield a cycle 1 population with a mean $=19.5$ under phenotypic selection, compared with a mean $=8.5$ predicted from the least squares estimate of 1.4.

Changes in genetic correlation estimates under selection also suggest that a factor with large effect was fixed during selection. The change in the $H$ and $C$ correlation estimates, with selection in the same direction for each trait, may be an example of the modified scheme 1 model described by Sheridan \& Barker (1974). This scheme describes a structured system composed of genes with large and small effects. Selection results in a slow change in the additive genetic covariance and a more rapid reduction in the additive genetic variances causing an increase in the absolute value of the genetic correlation. In this experiment, the correlation between $H$ and $C$ increased from 0.1 to 0.7 as the heritability dropped for each trait.

The $H$ and $L$ genetic correlation estimates are strong and positive for each cycle while selection for these two traits is in opposite directions. Selection in opposite directions on a gene with pleiotropic effects will tend to neutralize the net selection pressure and stabilize the genetic correlation. The $C$ and $L$ genetic correlation estimates move from negative to positive, also suggesting that a major factor responsible for a negative covariance was fixed during selection.

Defining flower colour in gerbera as a composite of three continuous variables provides an opportunity to apply the techniques of quantitative genetics to a breeding programme aimed at improving this character. However, if the assumption of many independent genes of equal effect is seriously violated genetic variance may be rapidly exhausted and the gain 
predictions overestimated. Evidence for this scenario is provided by CSA in this study. This also illustrates the need to estimate parameters in each selection cycle.

\section{Acknowledgements}

Fred Bliss and Hongzhan Huang provided many helpful comments on earlier manuscript versions. We also express our appreciation to Carlos Quiros. Some of the results of this paper were obtained by using the program package SAGE, which is supported by a U.S. Public Health Service Resource Grant (1 P41 RR03655).

\section{References}

AKAIKE, H. 1974. A new look at the statistical model identification. IEEE Trans. Aut. Control, AC-19, 716-723.

BAKER, L. H. AND CURNOW, R. N. 1969. Choice of population size and use of variation between replicate populations in plant breeding selection programs. Crop Sci., 9, 555-560.

BULMER, M. G, 1971. The effect of selection on genetic variability. Am. Nat., 105, 201-211.

BONNEY, G. E. 1984. On the statistical determination of major gene mechanisms in continuous human traits: regressive models. Am. J. Med. Genet., 18, 731-749.

BYRNE, T. G., HARDING J. AND NELSON, R. L. 1977. Greenhouse gerberas. Calif. Agric., 31, 21-22.

CANNings, C., Thompson, E. A. AND SKolnick, M. H. 1978. Probability functions on complex pedigrees. Adv. Appl. Prob., 10, 26-61.

CHEVALET, C. 1994. An approximate theory of selection assuming a finite number of quantitative trait loci. Génét. Sél. Évol, 26, 379-400.

COMSTOCK, R. E. AND RoBINSON, H. F. 1948. The components of genetic variance in populations of biparental progenies and their use in estimating the average degree of dominance. Biometrics, 4, 254-266.

CRow, J. F. AND kIMURA, M. 1970. An Introduction to Population Genetics Theory. Harper \& Row, New York.

DEMENAIS, F., LATHROP, M. AND LALOUEL, J. M. 1986. Robustness and power of the unified model in the analysis of quantitative measurements. Am. J. Hum. Genet., 38, 228-234.

EDWARDS, A. W. F. 1978. Likelihood. An Account of the Statistical Concept of Likelihood and its Application to Scientific Inference, 2nd edn. Cambridge University Press, London.
ELSTON, R. C. 1993. Some recent developments in the theoretical aspects of segregation analysis. In: P. P. Majumder (ed.) Human Population Genetics. Plenum Press, New York.

ELSTON R. C. AND STEWART, J. 1971. A general model for the genetic analysis of pedigree data. Hum. Hered., 21, 523-542.

FALCONER, D. S. 1989. Introduction to Quantitative Genetics, 3rd edn. Longman Scientific and Technical, New York.

GOMEZ-RAYA, L. AND BURNSIDE, E. B. 1990. The effect of repeated cycles of selection on genetic variance, heritability and response. Theor. Appl. Genet., 79, 568-574.

HARDING, J., BYRNE, T., HUANG, H. AND YU, Y. 1991. Multi-trait selection in flower crops. In: Harding, J., Singh F, and Mol, J. N. M. (eds.) Genetics and Breeding of Ornamental Species, pp. 157-178. Kluwer Academic, Boston.

HOSPTTAL, F. AND CHEVALET, C. 1993. Effects of population size and linkage on optimal selection intensity. Theor. Appl. Genet., 86, 775-780.

KHOURY, M. J, BEATY, T. H. AND COHEN, B. H. 1993. Fundamentals of Genetic Epidemiology. Oxford University Press, New York.

LERner, I. M. 1950. Population Genetics and Animal Improvement. Cambridge University Press, London.

McGUIRE, R. G. 1992: Reporting of objective color measurements. HortScience, 27, 1254-1255.

PESEK, J. AND BAKER, R. J. 1969. Desired improvement in relation to selection indices. Can. J. Plant Sci., 49, 803-804.

SAS InSTITUTE 1985. SAS User's Guide Basics, Version 5. SAS Institute, Cary, NC.

SAS INSTITUTE 1988. SAS/STAT User's Guide, Release 6.03. SAS Institute, Cary, NC.

SHERIDAN, A. K. AND BARKER, J. S. F. 1974. Two-trait selection and genetic correlation. II. Changes in genetic correlation during two-trait selection. Aust. J. Biol. Sci., 27, 89-101.

TOURJEE, K. R., HARDING, J. AND BYRNE, T. G. 1993. Colorimetric analysis of Gerbera flowers. HortScience, 28, 735-737.

TOURJEE, K. R., haRding, J. AND ByRNE, T. G. 1995. Complex segregation analysis of Gerbera flower colour. Heredity, 74, 303-310.

voss, D. H. 1992. Relating colorimeter measurement of plant color to the Royal Horticultural Society colour chart. HortScience, 27, 1256-1260.

WEBER G. AND WRICKE, W. E. 1986. Quantitative Genetics and Selection in Plant Breeding. W. de Gruyter, New York.

YU, Y., HARDING, J. AND BYRNE, T. 1993. Quantitative genetic analysis of flowering time in the Davis population of gerbera. II. Correlations with yield, flower quality traits and the efficiency of indirect selection. Euphytica, 70, 97-103. 